\title{
Representation of GaP Formation by a Reduced Order Surface Kinetics Model using P-Polarized Reflectance Measurements
}

\author{
S. Beeler ${ }^{\#}$ and H.T. Tran \\ Center for Research in Scientific Computation and \\ Department of Mathematics, Box 8205
}

\author{
N. Dietz \\ Department of Physics and \\ Department of Materials Science
}

\author{
North Carolina State University \\ Raleigh, NC 27695
}

\# E-mail: scbeeler@eos.ncsu.edu 


\section{Abstract}

The development of surface-sensitive real-time optical characterization techniques that are able to give insights into the surface reaction kinetics during organometallic deposition processes is essential for further progress in understanding and controlling thin film growth. It also will allow us to move the control point closer to the point where the growth occurs, which in a chemical beam epitaxy process is the surface reaction layer (SRL), built up of physisorbed and chemisorbed precursor fragments. This contribution presents results on the parameter estimation of rate constants and optical response factors in a reduced order surface kinetics (ROSK) model, which has been developed to describe the decomposition kinetics of the involved organometallic precursors and their incorporation in the film deposition. As a real-time characterization technique, we applied ppolarized reflectance spectroscopy (PRS) during low temperature growth of epitaxial GaP heterostructures on $\mathrm{Si}(001)$ substrates by pulsed chemical beam epitaxy (PCBE). The high surface sensitivity of PRS allows us to follow alterations in composition and thickness of the surface reaction layer (SRL) as they are encountered during periodic precursor supply. The linkage of the PRS response to the ROSK model provides the base for the parameter estimation of the reduced order surface kinetics model, giving insights into the organometallic precursor decomposition and growth kinetics. 


\section{INTRODUCTION}

Low pressure deposition methods, such as chemical beam epitaxy (CBE) and plasma enhanced chemical vapor deposition, play an important role in the manufacturing of nanostructure devices and advanced ULSI processing respectively. Areas of interest are novel quantum well electronic devices, improved densities of integrated electronic devices, methods of improving the control of epitaxial deposition to realize these devices, efficacy of resonant tunneling devices with respect to speed and reduced power, and demonstrated techniques for monolithic and hybrid integration of devices based on gallium arsenide (GaAs), indium phosphide (InP) ${ }^{1}$, and silicon ${ }^{2}$. Key targets in III-V compound/silicon heterostructures are the understanding and control of defect formation as well as the interactions and propagation of defects during later stages of compound heteroepitaxy growth. These are intimately linked to the understanding and control of the kinetics of heteroepitaxy, which in turn is closely related to the surface structure that depends on both reconstruction and the nature and distribution of defects in the epitaxial film. However, the progress in understanding and controlling thin film growth has been very slow, considering how little is known about chemical reaction pathways and reaction kinetics parameters during the decomposition process of the metal-organic (MO) precursors. Furthermore, the stringent tolerances in the engineering of advanced optoelectronic integrated circuits with respect to controlled thickness and composition of ultra-thin layers require the development of monitoring and control techniques that follow the deposition process with sub-monolayer resolution. These demands led to the development of surface-sensitive real-time optical sensors ${ }^{3}$ that are able to move the monitoring and control point close to the point where the growth occurs, which in a chemical beam epitaxy process is the surface reaction layer, built up of physisorbed and chemisorbed precursor fragments between the ambient and film interface. 
Applying optical probe techniques to real-time characterization of thin film growth inherits the challenge of relating surface chemistry processes, that drive the growth process, to growth/film properties, such as composition, instantaneous growth rate or structural layer quality. As illustrated in Figure 1, in deposition four primary regions are involved. Presently, most characterization techniques are being directed towards accurately measuring ambient process parameters, such as pressure, flux or temperature, since numerous probes are available to provide a relatively detailed assessment of the ambient. This strategy is clearly limited in its capability to deal with complex nonlinear surface chemistry processes, where the surface plays an integral role in the precursor decomposition pathways and small changes in the ambient composition can affect the growth substantially.

\section{Figure 1:}

The four primary regions involved in deposition are

(i) the ambient;

(ii) the surface reaction layer, which consists of species physisorbed or chemisorbed to the surface in dynamic equilibrium with both ambient and surface;

(iii) the surface itself; and

(iv) the near-surface region that can be defined as consisting of the outermost several atomic layers of the fabricated sample.

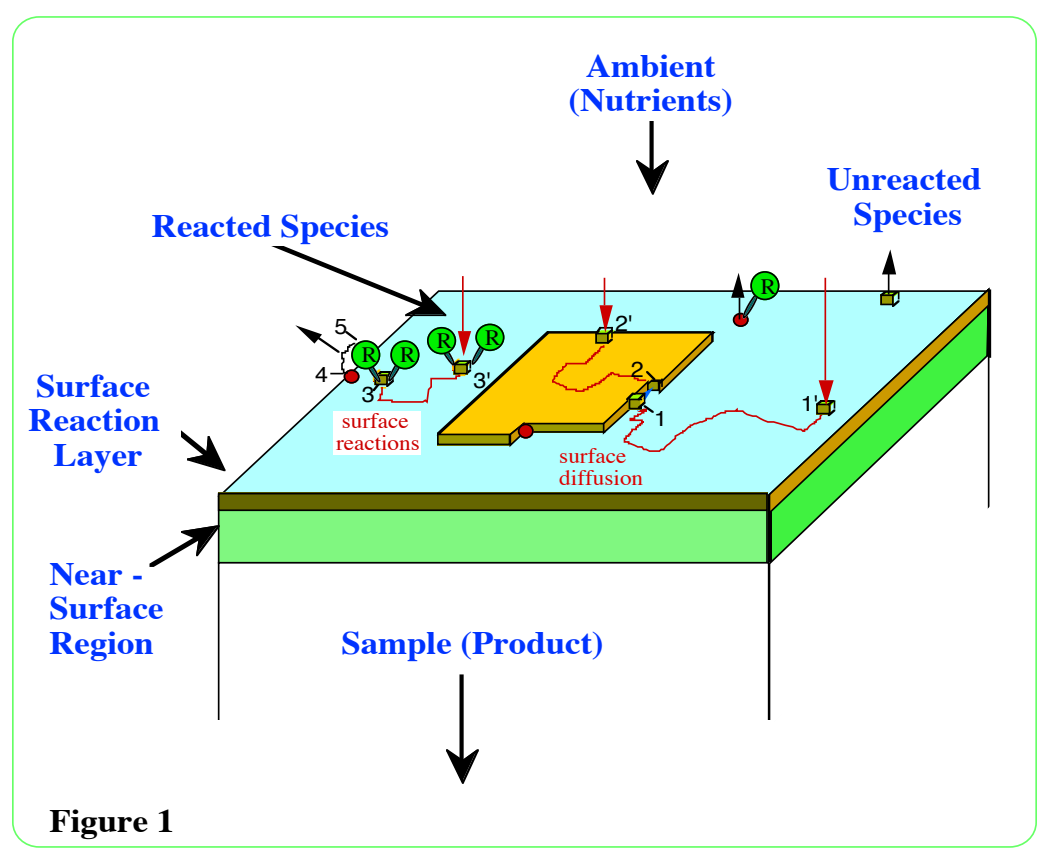


During the last few years, we developed and explored p-polarized reflectance spectroscopy $(\mathrm{PRS})^{4-6}$ as a highly surface-sensitive sensing technique, which allows us to follow the surface reaction kinetics under steady-state growth conditions. Utilizing this knowledge, we presently explore its application towards closed-loop control of deposition processes at low pressure $(\mathrm{PCBE})^{7}$.

First, in Section 2 we will give a brief background on the experimental growth and monitoring conditions and show results obtained by PRS during real-time characterization of heteroepitaxial growth of $\mathrm{GaP}$ on $\mathrm{Si}$ substrates. In Section 3 we introduce the model used to simulate the PRS measurements. We describe there the link of the PR response to the simulation parameters accessible through the reduced order surface kinetics (ROSK) model, which has been developed to describe the decomposition kinetics of the involved organometallic precursors ${ }^{7}$. The process of identifying these parameters is explained in Section 4, and Section 5 analyzes the results of the parameter identification. The knowledge gained from the ROSK model allows us to establish and validate surface reaction kinetics parameters, advancing our understanding of fundamental chemistry processes in thin film growth processes using organometallic precursors. Finally, our concluding remarks are contained in Section 6.

\section{EXPERIMENTAL SETUP AND RESULTS}

For monitoring both the bulk and surface properties during heteroepitaxial GaP growth on $\mathrm{Si}$, p-polarized reflectance spectroscopy (PRS) has been integrated in a pulsed chemical beam epitaxy (PCBE) system as schematically shown in Figure 2(b). In PCBE, the surface of the substrate is exposed to pulsed ballistic beams of TBP $\left[\left(\mathrm{C}_{4} \mathrm{H}_{9}\right) \mathrm{PH}_{2}\right]$ and TEG $\left[\mathrm{Ga}_{(}\left(\mathrm{C}_{2} \mathrm{H}_{5}\right)_{3}\right]$ at typically $350-450^{\circ} \mathrm{C}$ to accomplish nucleation and overgrowth of the silicon by an epitaxial $\mathrm{GaP}$ 
film. For PRS and laser light scattering (LLS) we employ p-polarized light beams at two angles of incidence (PR70: $\varphi=71.5^{\circ}$ and PR75: $\varphi=75.2^{\circ}$ ) using the wavelength $\lambda_{1}=632.8 \mathrm{~nm}$ and GlanThompson prisms, as illustrated in Figure 2(a). Further details on the experimental conditions are given in previous publications $s^{4-17}$.
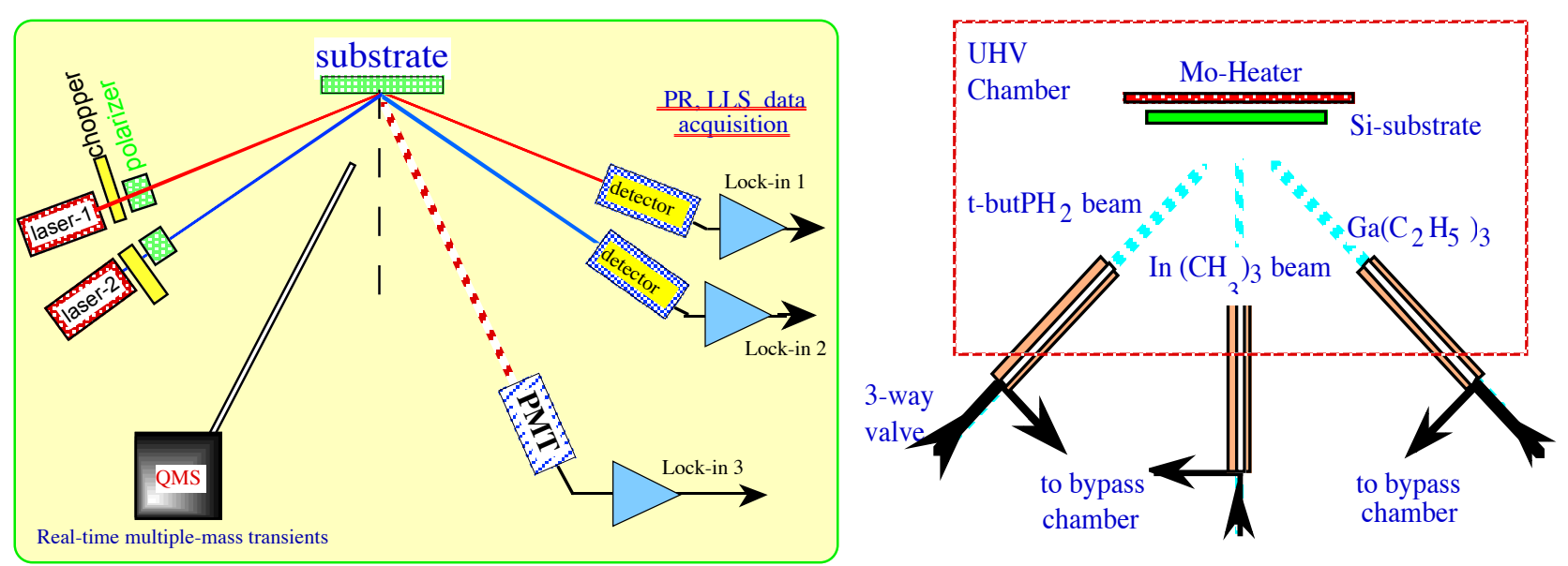

Figure 2: (a) Schematic setup of growth monitoring by PRS, LLS, and QMS; and (b) Setup of PCBE system for III-V compound semiconductor growth

During the preconditioning period, the PR signals change according to the temperature dependency of the substrate dielectric function. The PR signals are used to verify independent temperature measurements and to calibrate the actual surface temperature. A constant flow of Palladium purified $\mathrm{H}_{2}(10 \mathrm{sccm})$ is introduced to the growth chamber during the preconditioning as well as during the growth period. The background pressure in the growth system is $<1 \bullet 10^{-9}$ torr and increases to $5 \bullet 10^{-5}$ torr during pregrowth and to $2 \cdot 10^{-4}$ torr during growth.

Figure 3 shows the PR and LLS signals during heteroepitaxial growth of $\mathrm{GaP}$ on $\mathrm{Si}(001)$. After initiating growth at $1200 \mathrm{sec}$, minima and maxima are observed in the time evolution of the 
PR signals due to the interference phenomena as the film grows. It is noted that the maxima and minima of the two signals are inverted, which is due to the fact that one angle of incidence (PR75) is above - and the other (PR70) below - the pseudo-Brewster angle of the growing film. Superimposed on the interference oscillations of the reflectance is a fine structure that is strongly correlated to the time sequence of the supply of precursors employed during the steady-state growth conditions. The two insets in Fig. 3 show enlargements of the fine structure evolutions for $30 \mathrm{sec}$ of growth for PR75 and PR70, respectively.

\section{Figure 3:}

Monitoring of heteroepitaxial GaP growth under PCBE growth conditions by PRS and LLS. The insets show enlargements of the PR responses to periodically modulated SRL composition and thickness during pulsed precursor supply (see text).

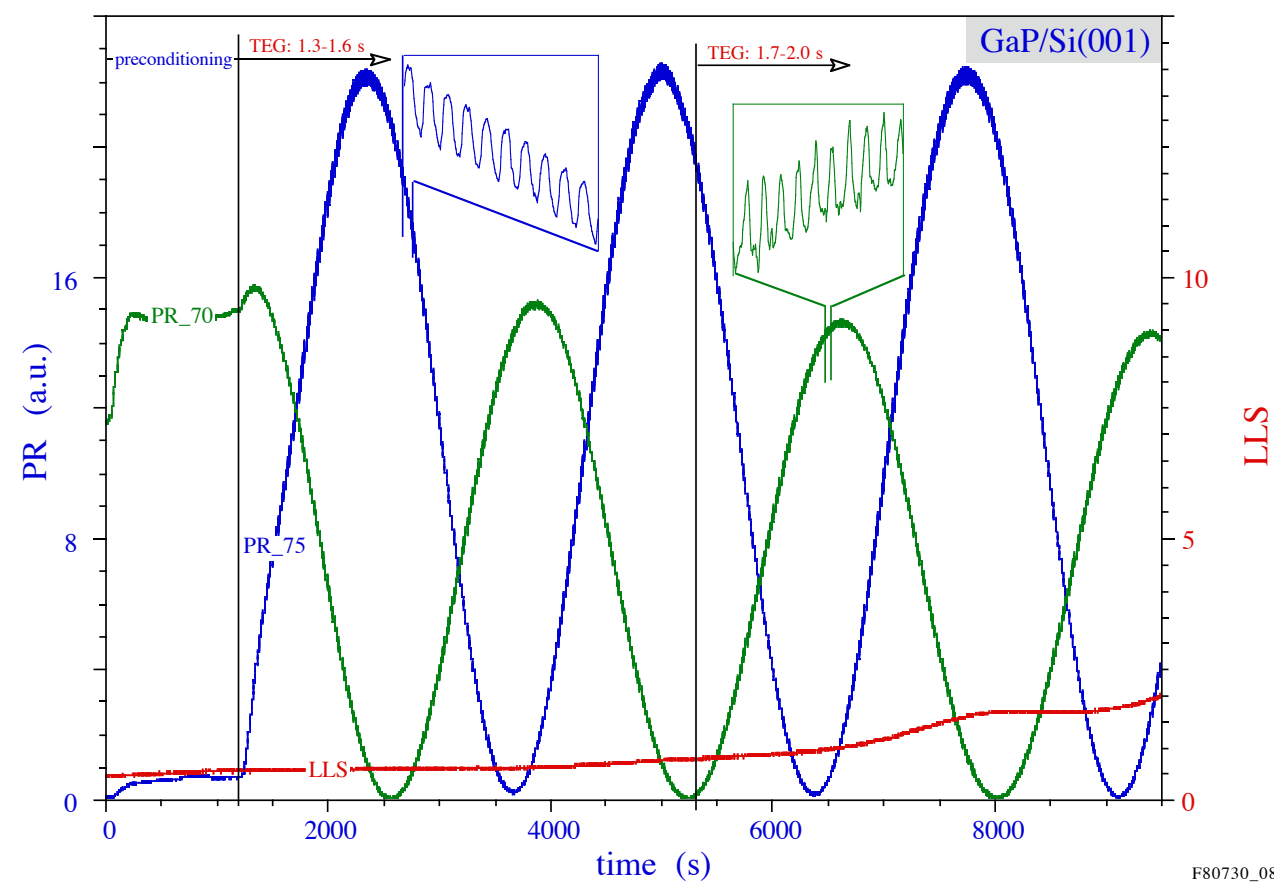

For the surface reaction kinetics analysis and the validation of performed simulations using the ROSK model presented in following sections, we varied two experimental parameters: (i) the position of the TEG pulse of $300 \mathrm{~ms}$ length within the precursor cycle sequence and (ii) the TEG flow rate. One growth condition was carried and monitored for at least 1-1/2 interference oscil- 
lations in order to get stable steady-state growth and to gather sufficient information to analyze and simulate the growth process.

\section{Figure 4:}

PR75 response to periodic exposure of growth surface to TBP and TEG precursor pulses, taken at the rising flank of an interference oscillation. The total cycle time is $3 \mathrm{sec}$ with TBP pulses from $0-0.8 \mathrm{sec}$ and TEG pulses from 1.3-1.6 sec.

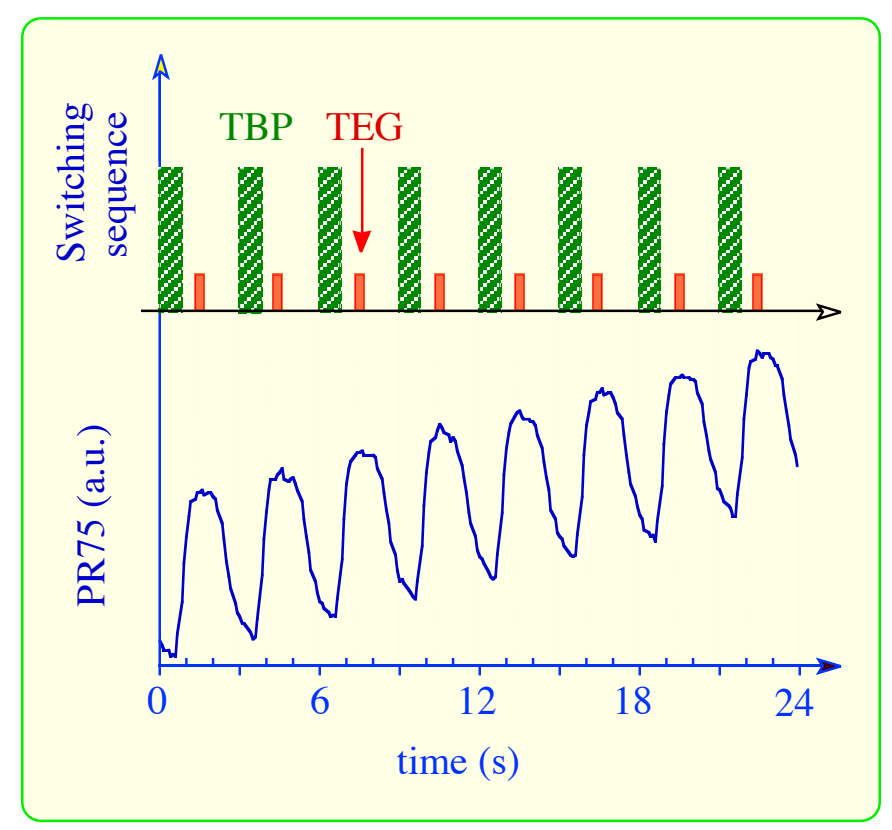

The correlation of the fine structure evolution with the pulsing sequence of the precursor supply is shown in more detail in Figure 4. In this figure, the PR response is taken during steadystate growth on a rising flank of an interference fringe using a pulse cycle sequence of $3 \mathrm{sec}$, a TBP pulse from 0.0-0.8 sec, a TEG pulse from 1.3-1.6 sec and continuous hydrogen flow during the complete sequence. In the first set of experiments, the flow rates and pulse durations of TBP (800 $\mathrm{ms}$ at $0.907 \mathrm{sccm}$, starting at $0.0 \mathrm{sec})$ and TEG $(300 \mathrm{~ms}$ at $0.04 \mathrm{sccm})$ were kept constant and only the start position of the TEG pulse was varied from $0.9 \mathrm{sec}$ up to $2.3 \mathrm{sec}$. The effect on the fine structure evolution is shown in Figure 5, where the start point of the TEG pulse is marked by an arrow. This influence of TEG pulse position on the PR response will be explained more fully in Section 5. For comparison, all PR responses are taken at the same inten- 
sity/reflectance level on a rising flank of an interference oscillation. We note that the exposure times as well as the precursor fluxes are identical for each trace shown in Fig. 5.

\section{Figure 5:}

PR75 responses for various TEG positions within a cycle sequence. The TBP exposure time, position and flux were kept constant. The flux and surface exposure time to TEG were constant; only the start point (marked by an arrow) was changed.

The TEG positions used were in steps of $0.2 \mathrm{~s}$ from (1) $0.9-1.2 \mathrm{sec}$ up to (8) 2.3-2.6 sec.

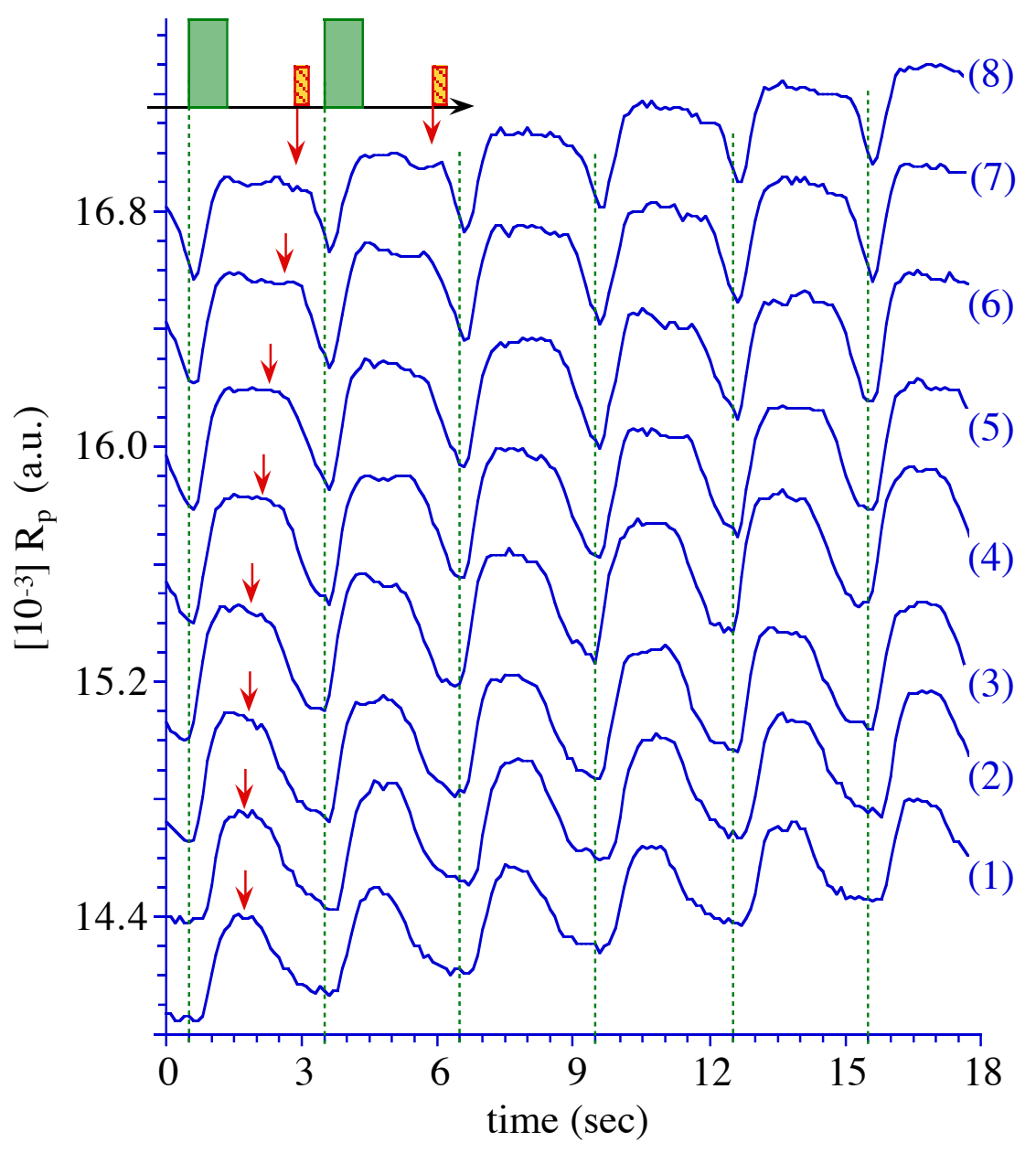

In the second set of experiments, the change in surface reaction kinetics and growth is evaluated for TBP:TEG flow ratios between 18 and 30. Figure 6 shows the PR and LLS signals during heteroepitaxial growth of $\mathrm{GaP}$ on $\mathrm{Si}(001)$ for three TEG flow settings $0.05,0.04$ and 0.03 sccm, with a TBP pulse of 0.0-0.8 sec (at $0.907 \mathrm{sccm}$ ) and a TEG pulse of 1.3-1.6 sec, in a pulse cycle sequence of $3 \mathrm{sec}$. With increasing TEG flow, the spacing of the interference oscillations widens according to a reduced growth rate. More details including comparisons with the results of simulations are given in section 5 . 
Figure 6:

PRS and LLS responses during heteroepitaxial $\mathrm{GaP}$ growth under PCBE growth, where at the marked positions the flow rate of TEG was changed, maintaining the pulse switching pattern for the supplied precursors.

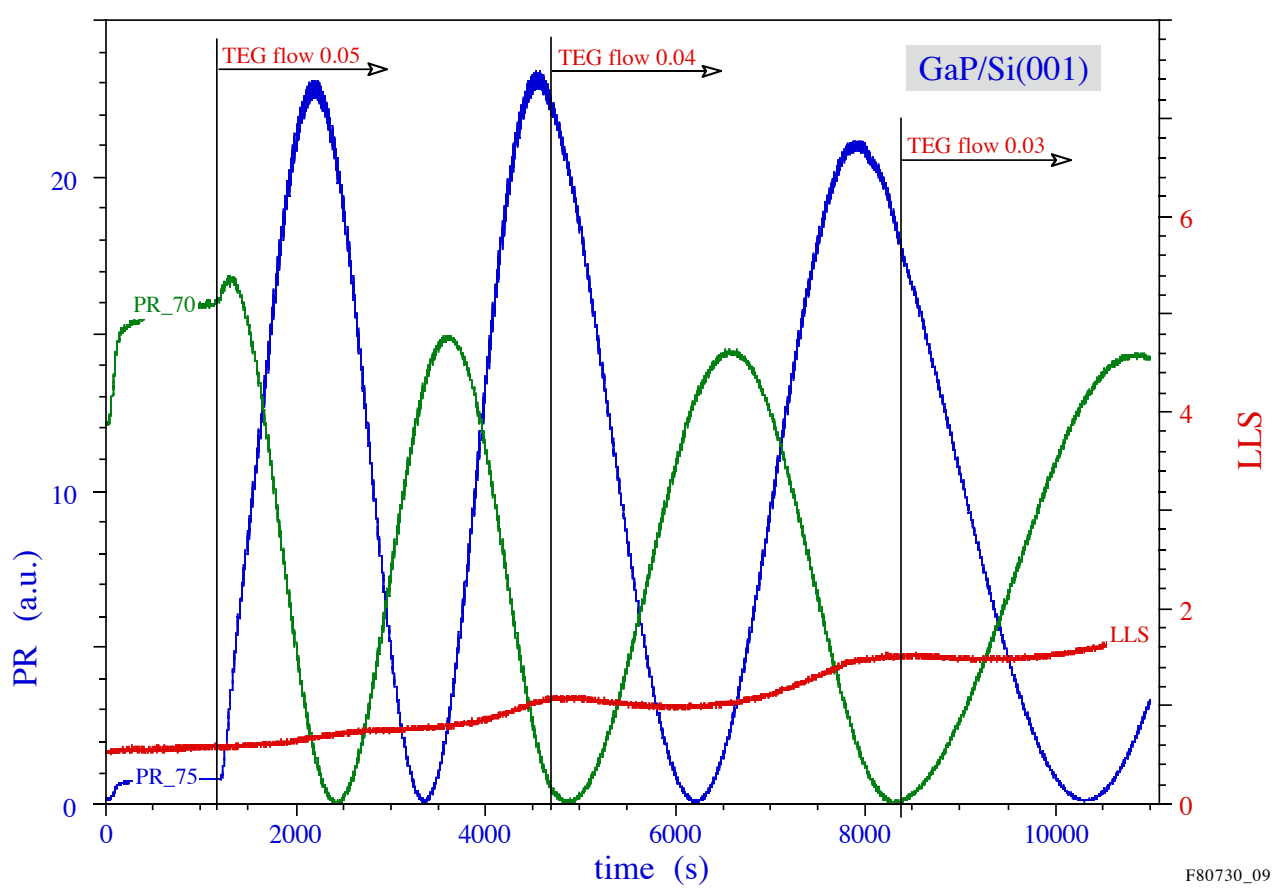

\section{MODELING OF PRS RESULTS}

We represent the structure of the chemical vapor deposition of a growing heteroepitaxial film with a four-layer medium model consisting of: (1) ambient - (2) surface reaction layer (SRL) (3) film - (4) substrate. We consider here GaP film growth on a Si substrate. The complex reflectivity coefficient for p-polarized incident light, given a four-layer stack, is ${ }^{18}$

$$
r_{p}=\frac{r_{12}\left(1+r_{23} r_{34} e^{-2 i \beta_{3}}\right)+\left(r_{23}+r_{34} e^{-2 i \beta_{3}}\right) e^{-2 i \beta_{2}}}{\left(1+r_{23} r_{34} e^{-2 i \beta_{3}}\right)+r_{12}\left(r_{23}+r_{34} e^{-2 i \beta_{3}}\right) e^{-2 i \beta_{2}}},
$$

where the Fresnel coefficients $\mathrm{r}_{\mathrm{k}(\mathrm{k}+1)}(\mathrm{k}=1,2$, and 3) for the interfaces 1-2, 2-3, and 3-4 are given by

$$
\mathrm{r}_{\mathrm{k}(\mathrm{k}+1)}=\frac{\varepsilon_{\mathrm{k}+1} \sqrt{\varepsilon_{\mathrm{k}}-\varepsilon_{1} \sin ^{2} \phi_{1}}-\varepsilon_{\mathrm{k}} \sqrt{\varepsilon_{\mathrm{k}+1}-\varepsilon_{1} \sin ^{2} \phi_{1}}}{\varepsilon_{\mathrm{k}+1} \sqrt{\varepsilon_{\mathrm{k}}-\varepsilon_{1} \sin ^{2} \phi_{1}}+\varepsilon_{\mathrm{k}} \sqrt{\varepsilon_{\mathrm{k}+1}-\varepsilon_{1} \sin ^{2} \phi_{1}}}
$$

and the phase shifts $\beta_{k}$ for the SRL $(\mathrm{k}=2)$ and the growing film $(\mathrm{k}=3)$ are given by 


$$
\beta_{\mathrm{k}}=\frac{2 \pi}{\lambda} \mathrm{d}_{\mathrm{k}} \sqrt{\varepsilon_{\mathrm{k}}-\varepsilon_{1} \sin ^{2} \phi_{1}} .
$$

Using equations (3.1)-(3.3), the reflectivity coefficient $\mathrm{r}_{\mathrm{p}}$ is a function of $d_{2}$ and $d_{3}$ (the thicknesses of the SRL and film respectively), $\varepsilon_{1}, \varepsilon_{2}, \varepsilon_{3}$ and $\varepsilon_{4}$ (the complex dielectric functions of the ambient, SRL, film and substrate respectively), and $\phi_{1}$ and $\lambda$. Here, $\phi_{1}$ denotes the angle of incidence and $\lambda$ is the wavelength of the impinging laser light ${ }^{18}$.

The values of $\varepsilon_{1}, \varepsilon_{3}, \varepsilon_{4}, \phi_{1}$ and $\lambda$ are constant in time, but $\varepsilon_{2}, \mathrm{~d}_{2}$ and $d_{3}$ vary in time as the film grows and the SRL composition and thickness change. To understand how these values change, we need a representative model of the chemical kinetics of the SRL, which approximates the pyrolysis of the primary source molecules as described by Bachmann et al. ${ }^{12}$. For TBP and TEG as source vapors forming GaP, we employed a reduced order surface kinetics (ROSK) ${ }^{7}$ model. The ROSK model makes the simplifying assumption that the many reactions which make up the TBP pyrolysis are combined into one step, the reactions which make up the TEG decomposition are combined into two steps, and the formation of $\mathrm{GaP}$ is one final step. The process is driven by a periodic source vapor cycle as described in the last section.

Thus the kinetic model representing the SRL reactions is given by the following system of ordinary differential equations:

$$
\begin{aligned}
& \frac{\mathrm{d}}{\mathrm{dt}} \mathrm{n}_{1}(\mathrm{t})=\mathrm{S}_{1}(\mathrm{t})-\mathrm{k}_{1} \mathrm{n}_{1}(\mathrm{t})-\mathrm{k}_{\mathrm{GaP}} \mathrm{n}_{1}(\mathrm{t}) \mathrm{n}_{3}(\mathrm{t}) \frac{1}{10^{-8} \mathrm{~mol}} \\
& \frac{\mathrm{d}}{\mathrm{dt}} \mathrm{n}_{2}(\mathrm{t})=\mathrm{S}_{2}(\mathrm{t})-\mathrm{k}_{2} \mathrm{n}_{2}(\mathrm{t})-\mathrm{k}_{3} \mathrm{n}_{2}(\mathrm{t}) \\
& \frac{\mathrm{d}}{\mathrm{dt}} \mathrm{n}_{3}(\mathrm{t})=\mathrm{k}_{3} \mathrm{n}_{2}(\mathrm{t})-\mathrm{k}_{4} \mathrm{n}_{3}(\mathrm{t})-\mathrm{k}_{\mathrm{GaP}} \mathrm{n}_{1}(\mathrm{t}) \mathrm{n}_{3}(\mathrm{t}) \frac{1}{10^{-8} \mathrm{~mol}} \\
& \frac{\mathrm{d}}{\mathrm{dt}} \mathrm{n}_{4}(\mathrm{t})=\mathrm{k}_{\mathrm{GaP}} \mathrm{n}_{1}(\mathrm{t}) \mathrm{n}_{3}(\mathrm{t}) \frac{1}{10^{-8} \mathrm{~mol}}
\end{aligned}
$$


The variables $\mathrm{n}_{1}, \mathrm{n}_{2}$ and $\mathrm{n}_{3}$ represent the number of moles of the components of the SRL: active surface phosphorus fragments, diethylgallium (DEG), and monoethylgallium (MEG) and active gallium fragments respectively. In the first equation the change in active phosphorus fragments is written as a sum of a source term $S_{1}$, a desorption loss term $-k_{1} n_{1}$ and a reaction term forming GaP. The second equation, which describes the defragmentation of TEG, contains a source term $S_{2}$, a desorption loss term $-k_{2} n_{2}$ and a term of decomposition into MEG and active gallium fragments. The third equation (change in MEG and active surface gallium fragments) has a term of creation, a desorption loss term and a reaction term forming GaP. The fourth variable, $n_{4}$, represents the numbers of moles of created GaP integrated in the deposited GaP film layer. This equation contains only the single reaction term for the formation of $\mathrm{GaP}$ from active surface $\mathrm{Ga}$ and $\mathrm{P}$ and has to account also for any surface activation processes.

The source terms in the differential equations are based on the source vapor pulses. More specifically, we modeled the source terms by the following expression:

$$
\mathrm{S}_{1}(\mathrm{t})=\frac{\mathrm{P}_{1}(\mathrm{t}) \gamma \beta_{\mathrm{TBP}}}{\mathrm{V}_{\mathrm{TBP}}},
$$

where $P_{1}(t)$ is the source vapor flow rate. $V_{T B P}$ is the molar volume of TBP and the constant $\beta_{T B P}$ is the sticking coefficient of TBP. The geometrical parameter $\gamma$ represents how much of the source vapors actually hit the surface of the wafer (a constant dependent on the structure of the reactor). Similarly, the second source term is represented by:

$$
\mathrm{S}_{2}(\mathrm{t})=\frac{\mathrm{P}_{2}(\mathrm{t}) \gamma \beta_{\mathrm{TEG}}}{\mathrm{V}_{\mathrm{TEG}}}
$$

with corresponding $P_{2}(t), V_{T E G}$ and $\beta_{T E G}$ for the TEG pulse, and the same constant $\gamma$. For each source term we are using a constant flow rate between start and stop times (and zero flow else- 
where), as described in Section 2. There is a small time difference between the switching on (or off) of the pulse and the start (or stop) of the source vapors at the surface. This is caused by the time needed for the source vapor gates to open or close and for the vapors to travel to the surface. We account for it with a parameter delay, so that for a source vapor pulse set to start at $t_{\text {on }}$ and stop at $t_{\text {off }}$, the source vapors will actually reach the surface starting at $t_{o n}+$ delay and stopping at $t_{\text {off }}+$ delay. The delay was estimated to be $0.72 \mathrm{sec}$, using a parameter indentification process described in the next section.

The system of differential equations (3.4)-(3.7), together with the source terms (3.8) and (3.9) and appropriate initial conditions, can be solved numerically for the number of moles $n_{1}, n_{2}, n_{3}$ and $n_{4}$. From these solutions, the film and SRL thicknesses are found by the following equations:

$$
\begin{aligned}
& \mathrm{d}_{3}(\mathrm{t})=\frac{\mathrm{V}_{\mathrm{GaP}}}{\mathrm{A}} \mathrm{n}_{4}(\mathrm{t}) \\
& \mathrm{d}_{2}(\mathrm{t})=\frac{\alpha_{\text {SRL }}}{\mathrm{A}}\left[\mathrm{V}_{1} \mathrm{n}_{1}(\mathrm{t})+\mathrm{V}_{2} \mathrm{n}_{2}(\mathrm{t})+\mathrm{V}_{3} \mathrm{n}_{3}(\mathrm{t})\right],
\end{aligned}
$$

and the effective dielectric function of the SRL is given by the formula

$$
\varepsilon_{2}(t)=1+\left[\frac{n_{1}(t)}{\sum_{k=1}^{3} n_{k}(t)} F_{1}+\frac{n_{2}(t)}{\sum_{k=1}^{3} n_{k}(t)} F_{2}+\frac{n_{3}(t)}{\sum_{k=1}^{3} n_{k}(t)} F_{3}\right],
$$

which is derived from the Sellmeier equation ${ }^{19}$. In the above three equations, $\mathrm{A}$ is the surface area of the wafer, the values $V_{k}$ are the molar volumes of the components $n_{k}$, and $V_{G a P}$ is the molar volume of GaP. The parameters $F_{k}$ are the optical responses of the components of the SRL and $\alpha_{S R L}$ is an effective SRL thickness parameter representing the percentage of the SRL 
that contributes to the reflectance behavior. With the values of the temporal dependent parameters $\varepsilon_{2}, \mathrm{~d}_{2}$ and $\mathrm{d}_{3}$ found by equations (3.10)-(3.12), and with the constant parameters $\varepsilon_{1}, \varepsilon_{3}, \varepsilon_{4}, \phi_{1}$

and $\lambda$, the reflectivity coefficient $r_{p}$ can be computed from equations (3.1)-(3.3). From $r_{p}$, we then find the value that is actually measured in the experiments by computing the reflectance $\mathrm{R}_{\mathrm{p}}=\left|\mathrm{r}_{\mathrm{p}}\right|^{2}$

In the equations described in this section, $\phi_{1}, \lambda, \varepsilon_{1}, V_{1}, V_{2}, V_{3}, V_{G a P}, V_{T B P}, V_{T E G}, A, \beta_{1}, \beta_{2}$, $\alpha_{\mathrm{SRL}}$, all start/stop times, and flow rates contributing to $P_{1}$ and $P_{2}$ are known quantities. The values of the dielectric functions $\varepsilon_{3}$ and $\varepsilon_{4}$, the rate constants $k_{1}, k_{2}, k_{3}, k_{4}$ and $k_{G a P}$, the optical responses $F_{1}, F_{2}$ and $F_{3}$, the geometrical parameter $\gamma$, and delay are not known. Our work in the following sections is to find values of these parameters so that the mathematical model most closely matches experimental results.

\section{PARAMETER IDENTIFICATION PROBLEM}

In this section we formulate the inverse least squares problem used to find the set of parameters with which the results of the mathematical model of the reflectance (described in Section 3) match most closely with the experimental data. More specifically, we are looking for the vector of parameters $\overrightarrow{\mathrm{q}}=\left(\mathrm{F}_{1}, \mathrm{~F}_{2}, \mathrm{~F}_{3}, \mathrm{k}_{1}, \mathrm{k}_{2}, \mathrm{k}_{3}, \mathrm{k}_{4}, \mathrm{k}_{\mathrm{GaP}}, \gamma\right.$, delay $)$ that minimizes the following cost function:

$$
J(\vec{q})=\sqrt{\sum_{i}\left(R_{\exp }\left(t_{i}\right)-R_{\text {calc }}\left(t_{i}, \vec{q}\right)\right)^{2}}
$$


Here $R_{\text {exp }}\left(t_{i}\right)$ is the experimental data set at the measurement times $t_{i}$ and $\mathrm{R}_{\text {calc }}\left(\mathrm{t}_{\mathrm{i}}, \overrightarrow{\mathrm{q}}\right)$ is the simulation results calculated at the same times using the parameter set $\vec{q}$.

We do not include $\varepsilon_{3}$ and $\varepsilon_{4}$ in $\vec{q}$, because larger numbers of parameters make the minimization process increasingly difficult. We can remove these two parameters from the above parameter estimation problem by formulating a separate but simpler estimation problem. In particular, we use a three-layer stack as a simpler model of the growing film: removing the SRL from consideration leaves just the ambient, film and substrate layers. The formula for calculating the reflectance for a three-layer stack analytically is given by:

$$
r_{3, p}=\frac{r_{13}+r_{34} e^{-2 i \beta_{3}}}{1+r_{13} r_{34} e^{-2 i \beta_{3}}}
$$

where $r_{13}$ and $r_{34}$ are Fresnel coefficients for the reflection from interfaces 1-3 and 3-4 (now that layer 2 is removed), and the phase shift $\beta_{3}$ is for the film layer. These values are calculated by formulas analogous to (3.2) and (3.3). 


\section{Figure 7:}

Extraction of envelope reflectance spectra from PR75 data by removing the fine structure, shown near a turning point (see text for detail).

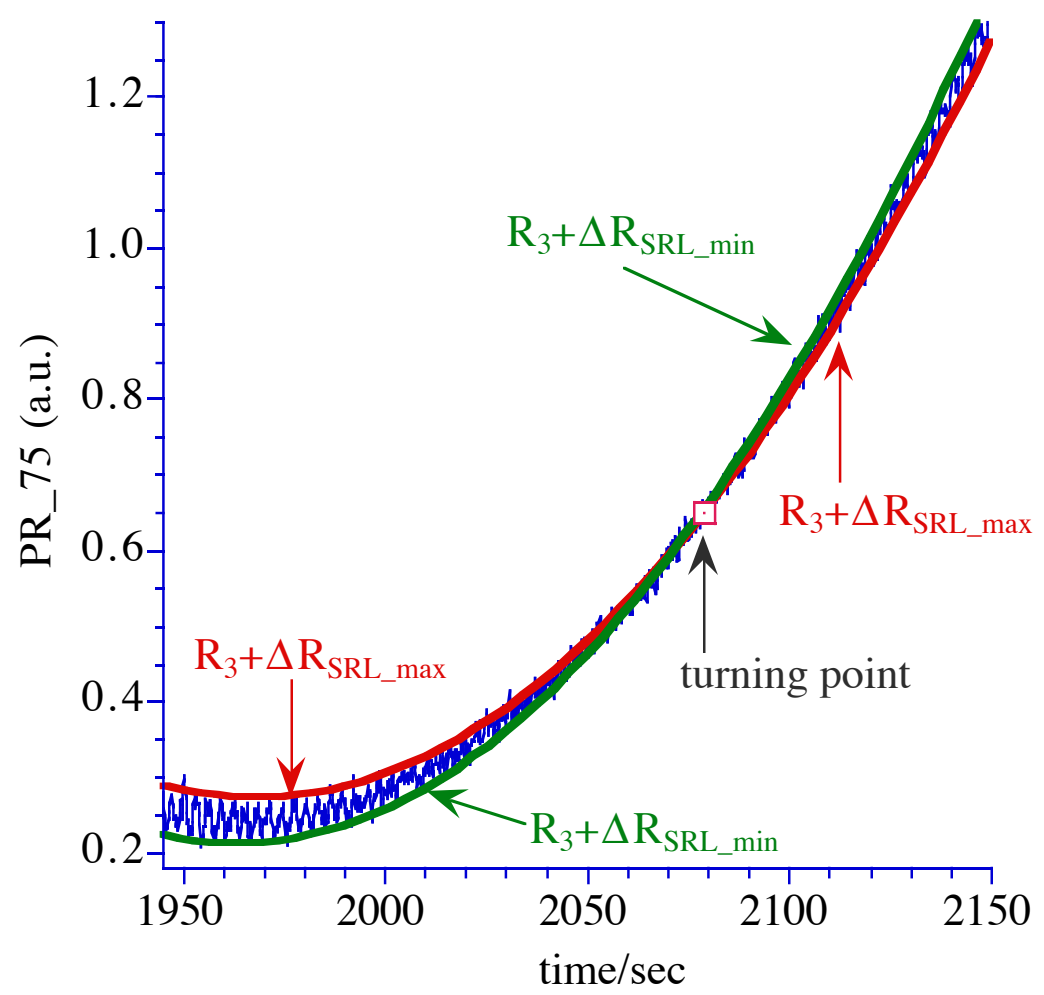

To compare results from this formula with experimental results, we first remove the effects of the SRL from the experimental data by removing the small-amplitude fine structure oscillations modulated with the precursor cycle from the large-amplitude interference oscillations, which have a periodicity of several hundreds of seconds. In order to remove the fine structure, first the curves on either side of the data forming an envelope around it must be found. The experimental version of the three-layer stack reflectance is then found by switching from one side of the envelope to the other where the fine structure "turns" from positive to negative (from adding to the three-layer stack reflectance to subtracting from it) or vice versa. This orientation of the fine structure is cyclical with the interference oscillations, either turning twice per oscillation or else not turning at all, in which case there is no switching between envelope sides. Figure 7 shows this extraction of the three-layer reflectance out of experimental data near a turning point. The 
three-layer reflectance plus the minimal influence from the SRL during a cycle is shown on one side of the data (switching sides at the turning point), while the other side represents the threelayer stack plus the maximal influence from the SRL during the cycle.

With this method of extracting the experimental three-layer stack reflectance $R_{3 \text {,exp }}$, we can identify the parameters $\varepsilon_{3}$ and $\varepsilon_{4}$, as well as an average growth rate $g_{r}$ (used to find the film thickness at the times $t_{i}$ ), by comparing the calculated reflectance $\mathrm{R}_{3 \text {,calc }}=\left|\mathrm{r}_{3, \mathrm{p}}\right|^{2}$ from equation (4.2) to $R_{3, \exp }$. This is done also through an inverse least squares formulation by finding $\overrightarrow{\mathrm{r}}=\left(\varepsilon_{3}, \varepsilon_{4}, \mathrm{~g}_{\mathrm{r}}\right)$ that minimizes the cost function

$$
J(\vec{r})=\sqrt{\sum_{i}\left(R_{3, \exp }\left(t_{i}\right)-R_{3, \text { calc }}\left(t_{i}, \vec{r}\right)\right)^{2}} .
$$

Once the values of $\varepsilon_{3}$ and $\varepsilon_{4}$ are found, they can be used in solving the four-layer stack parameter identification problem to find the unknown parameters $F_{1}, F_{2}, F_{3}, k_{1}, k_{2}, k_{3}, k_{4}, k_{G a P}, \gamma$, and delay.

\section{ANALYSIS OF RESULTS}

Comparing measurements taken with the TEG pulse position varied while all other conditions are fixed (see e.g., the fine structure shown in Fig. 5) reveals several important characteristics in the fine structure. We will explain these features and show how the mathematical simulation of the growth process, using the reduced order surface kinetics model, replicates these features.

Looking at the fine structure (in the PR75 data), the most noticeable change with the TEG pulse position variation is the starting position of the downward slope (near marked arrows in the 
Fig. 5) which is present in every data set but moves to later in the cycle as the TEG pulse moves to later in the cycle. This start of the downward slope, which is the only feature so dependant on the TEG pulse placement, clearly relates to the source TEG, the subsequent TEG defragmentation, and active gallium attachment on the surface.

In contrast, the starting position of the upward slope in the fine structure remains in the same place shortly after the start of the cycle, independently of the TEG pulse position. It can be related to the source TBP exposure, its defragmentation, and the formation of active phosphorus on the surface. Both starting positions are delayed by approximately $0.72 \mathrm{~s}$ after the start of the pulses. This delay is due to the time needed to open the source vapor gates and the time for the vapors to travel to the surface, as noted in the description of the model. 


\section{Figure 8:}

Simulated PR75 responses for various TEG positions within a cycle sequence, with the rest of the source cycle properties kept constant. These properties and the location shown match those of the experimental data in Fig. 5. The changed TEG starting point is marked by an arrow.

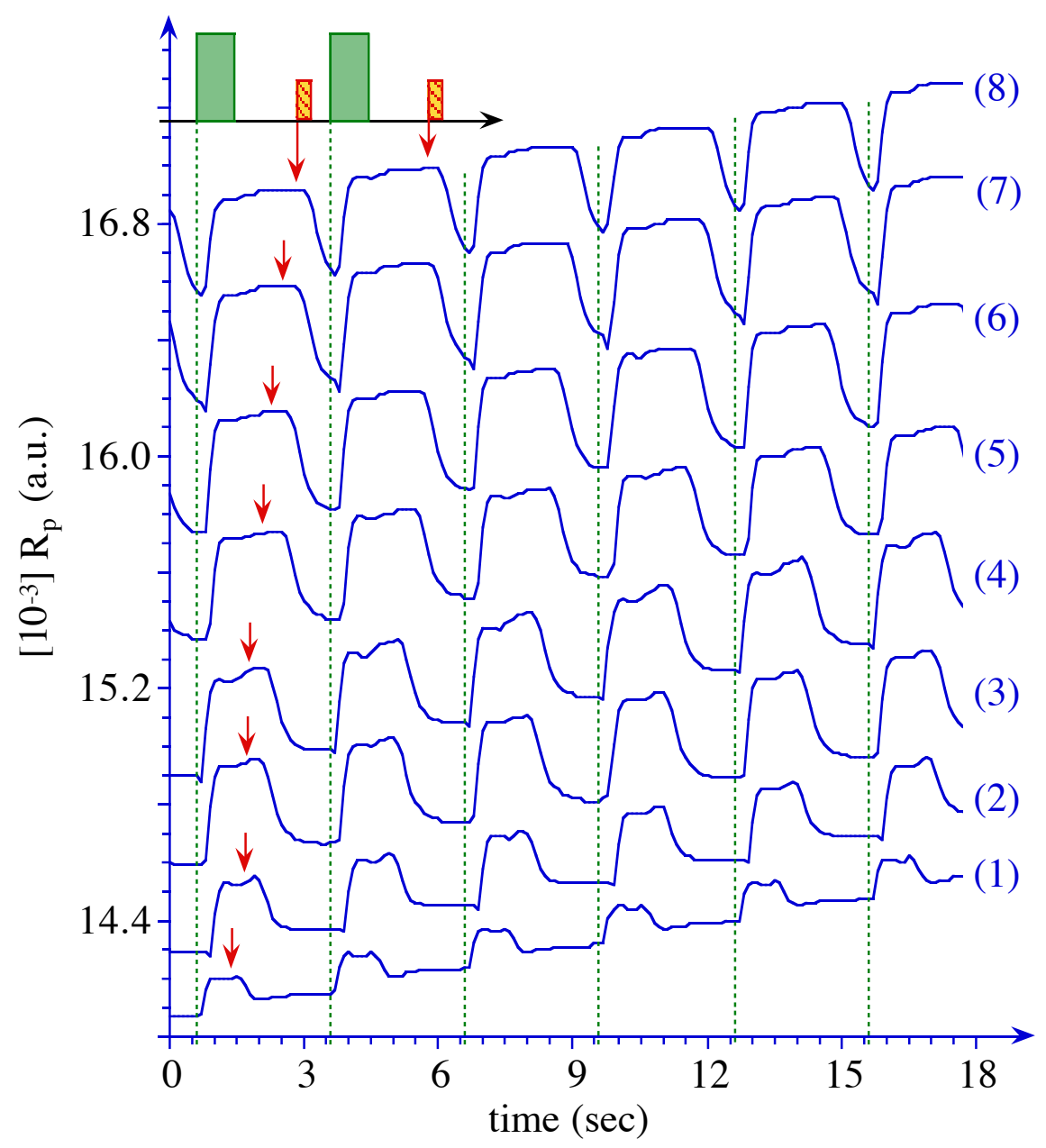

The same upward and downward slopes, and delay characteristics, can be seen in Figure 8, where the fine structure evolutions of the simulated data are compared at the same points as the experimental data in Fig. 5. The gap between the downward and upward slopes can be analyzed by the FWHM (Full-Width Half-Maximum), defined by the width between times on the down and up slopes with values halfway between the maximum and minimum reflectance during that cycle. Figure 9 illustrates how this width shrinks as the TEG pulse is moved toward the end of the cycle and closer to the next TBP pulse. This change, in both the experimental and calculated data, follows the pulse position nearly linearly.

The fine structure amplitude (the difference between maximum and minimum reflectance over a cycle) also changes slightly but clearly with the change in TEG pulse position. As shown in 
Figure 9, the amplitude is largest for TEG pulses near the middle of the range used. This can be explained as a result of the closeness of the TEG and TBP pulses. If the TEG is input soon after the TBP, there will be a large GaP formation reaction, leaving little or no active gallium to carry over to the next cycle. If the TEG comes in very late in the cycle, right before the next TBP pulse, there may not be time for the decomposition of all the TEG to gallium to occur before the GaP formation with the incoming phosphorus starts. With a more central TEG pulse, the phosphorus and gallium will each have the time to build up on the surface, in turn creating more extreme changes in the SRL thickness and composition and therefore larger fine structure amplitude.

Figure 9:

Properties of the PR responses as affected by the TEG start position:

(a) average film growth rate,

(b) amplitude of the fine structures in Figs. 5 and 8, and

(c) FWHM of the fine structures.

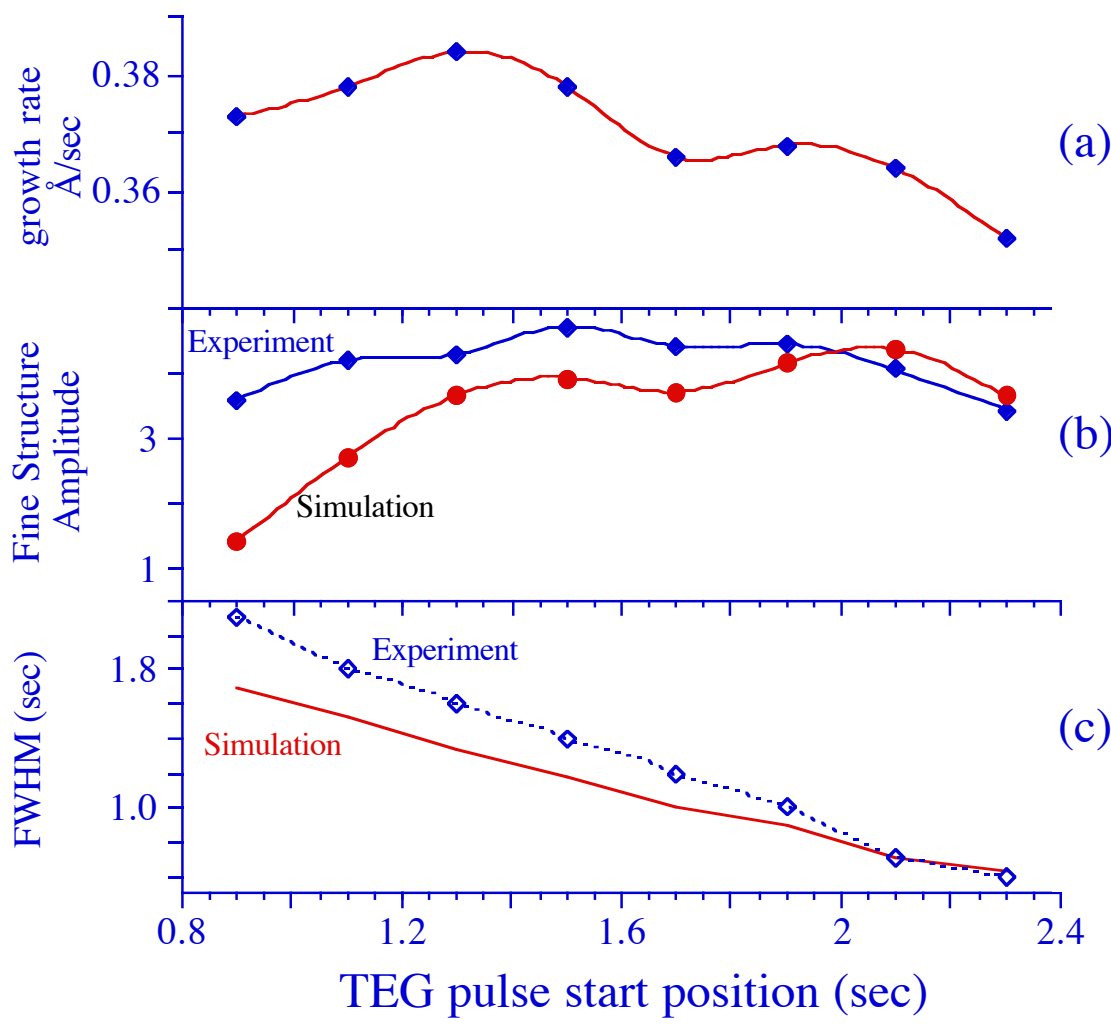

(a) 
Note that this analysis of the fine structure is at a specific place on the interference oscillations, fairly high on a rising flank. Other places, particularly on the other side of a turning point, will have different characteristics (for example, the TEG pulse may result in a jump upward and the TBP pulse in a jump downward).

One larger-scale feature of the reflectance data we can look at is the average film growth rate for the various TEG pulse positions, as shown in Fig. 9. The general downward slope can be explained in terms of the closeness of the two pulses. As the TEG pulse moves later in the cycle away from the TBP pulse there is less phosphorus to react with, so there is more active gallium left on the surface to be lost via desorption. The TEG pulse positions nearest the start of the cycle seem to be too close to the TBP pulse for the fastest growth rate, however. The incoming TEG and its defragmentation products may be partially blocked from the available active phosphorus in the SRL by TBP that failed to stick and/or desorbed phosphorus that is sitting loose on the surface.

Another large-scale characteristic feature of the data sets is the position of (or complete lack of) turning points in the fine structure. These come in pairs for every interference oscillation or not at all, as discussed last section (with a close-up of a turning point in Fig. 7). The turning point positions can be characterized by the derivative of the reflectance. The overall derivative amplitude is related to the periodic thickness changes in the SRL. This amplitude is minimized at the turning points, where the fine structure amplitude is smallest and so the reflectance curve is least steep. Figure 10 shows the close match between the experimental and calculated derivative amplitudes and turning point positions. In earlier works ${ }^{5,6}$, we showed that the locations of these turning points change as a function of the SRL dielectric properties. The good agreement shown in Figure 10 indicates that the SRL dielectric properties were obtained correctly. 


\section{Figure 10:}

Characterization of fine structure envelope using the first derivative of the reflectance spectrum. For comparison, the experimental and simulated reflectance spectra with their (numerical) first derivatives are shown. (TEG pulse is $1.3-1.6 \mathrm{~s}$.)

The simulated reflectance spectrum lies on top of the experimental spectrum and is not shown for clarity.

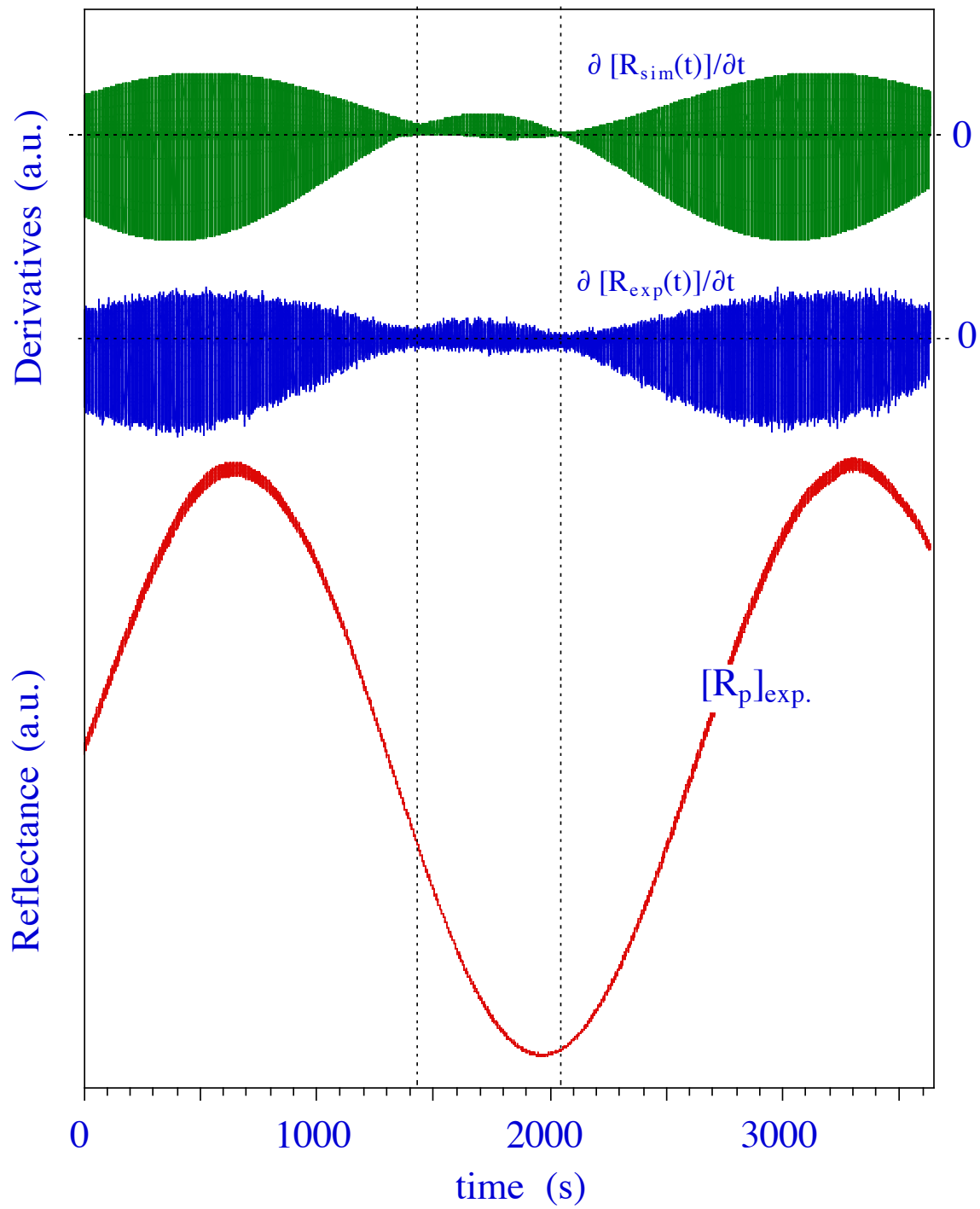

The measurements taken with the TEG pulse position fixed but the flow rate varied also correspond to what is expected. Examples of the fine structure (again for PR75) for the three TEG flow rates are shown in Figure 11, for both experimental data and simulated data. In contrast with the variation of the pulse position, here the shape of the fine structure remains the same, since the shape of the source vapor cycle is the same. The positions of changes in the slope remain constant due to the constant position of the TEG pulse. The amplitude of the fine structure 
does change, since as the TEG flow rate increases there will be more gallium deposited in the SRL, and this will cause a larger effect in the reflectance. Larger TEG flow also results in a much faster film growth rate, which causes steeper large-scale curves as seen in Fig. 11 and faster interference oscillations as seen in Fig. 6. Both the experimental and simulated data sets show these characteristics and both results agree with each other extremely well.

\section{Figure 11:}

Experimental and simulated PR75 responses for various TEG fluxes under steadystate growth conditions. The TBP exposure time, position and flux were kept constant, as were the surface exposure time and pulse position for TEG.

The TEG fluxes used were:

(1) $0.05 \mathrm{sccm}$

(2) $0.04 \mathrm{sccm}$

(3) $0.03 \mathrm{sccm}$

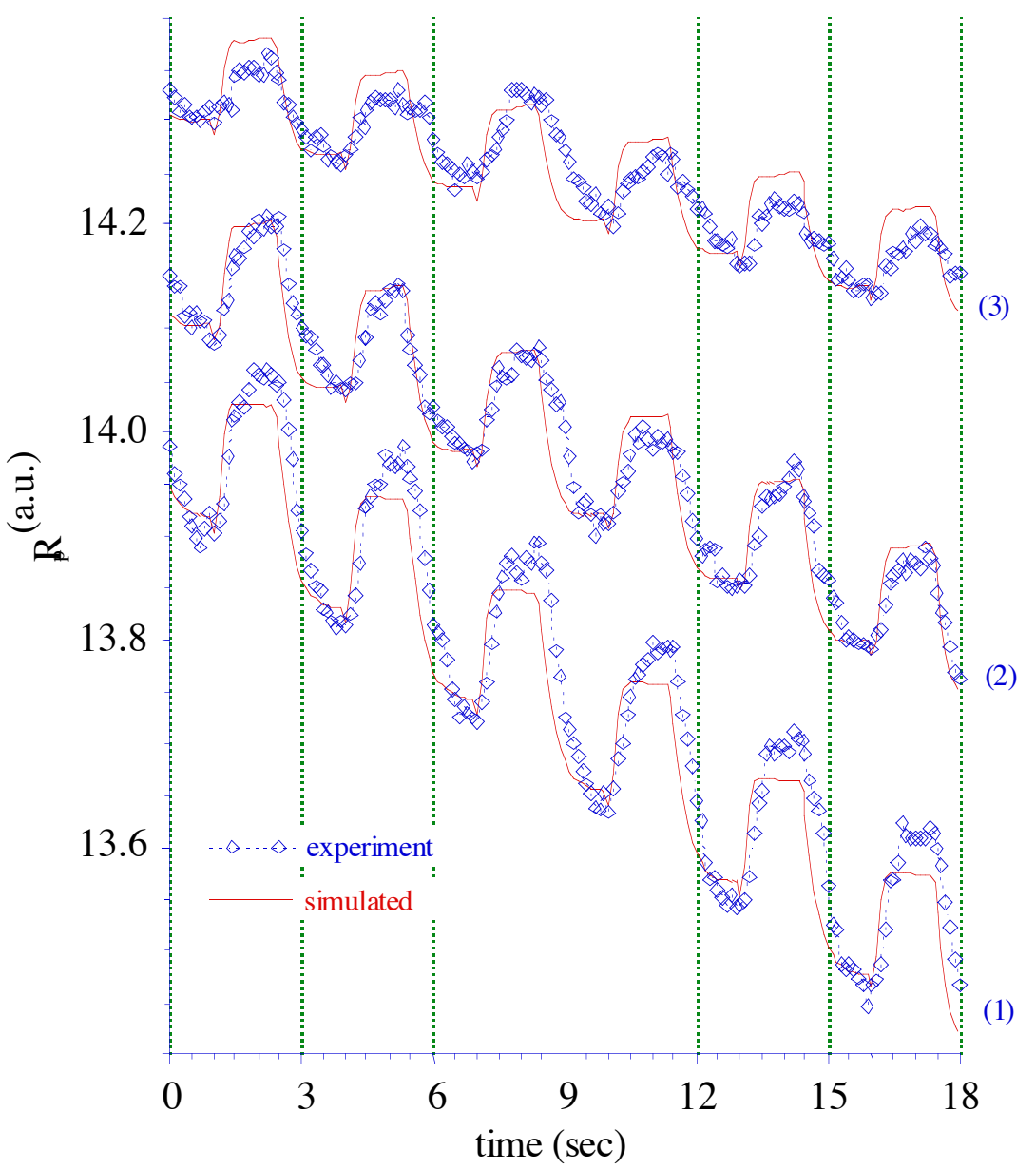

The steps in the generation of a set of simulated data which were used to compare against experimental data presented in Figure 3 are shown in detail in Figure 12, for the TEG pulse 1.3-1.6 sec (and TBP pulse 0.0-0.8 sec). The three SRL components are the result of the source pulses 
and the ROSK model simulation. From the SRL components, the SRL thickness and dielectric function are found. These values then contribute to the calculated reflectance. Fig. 12 shows how the arrival of gallium in the SRL causes the downward slope in the fine structure and how the arrival of phosphorus causes the upward slope. The good fit of this simulated fine structure to the experimental data as shown in Fig. 12 will also hold for the rest of the interference oscillations. This is illustrated in Fig. 10, where the reflectance derivatives match, as the actual reflectance curves match (and the fine structure amplitudes and turning points also agree). The closeness of the fit and the correlation of the significant features discussed above support the ROSK model of the growth process and its effects on the reflectance measurements.

An important aspect of the behavior of the SRL kinetics which can be seen in Fig. 12 is the difference between a phosphorus- and gallium-terminated surface at the end of a cycle sequence. We had at first expected a phosphorus-terminated surface at the end of each cycle sequence time, where the TEG pulse is almost completely used up through desorption or formation of GaP, leaving some phosphorus in the SRL at the start of the next pulse cycle. However, simulated reflectance data with this type of behavior could not fit the experimental data. Instead a set of parameters which resulted in a gallium-terminated surface (where the TEG pulse is not all used up during at the end of the cycle time, leaving an amount of gallium in the SRL carrying over to the next cycle) gave a much more accurate fit as described above.

The data measured at the second angle (PR70) has structure and features similar to the PR75 data, with the major difference being the inversion of interference oscillation maxima/minima since the angles are on opposite sides of the pseudo-Brewster angle. Analysis of these measurements using the same model results in parameters similar to those found for PR75 (which are given below) and a similar fit of the reflectance data. There are a few noticeable differences 
between the two, which can be explained by the measurements being taken with light beams hitting different points on the surface. If the growth is somewhat uneven this could cause differences in the parameters in the growth model when the two data sets are compared.

Figure 12:

Contributions of parts of the model to the simulated reflectance:

(a) Source vapor fluxes,

(b) Number of moles of the three SRL components,

(c) SRL thickness, and

(d) SRL effective dielectric function real and imaginary parts.

These result in:

(e) Simulated PR response, shown fitting experimental measurements (for TEG $1.3-1.6 \mathrm{sec})$.

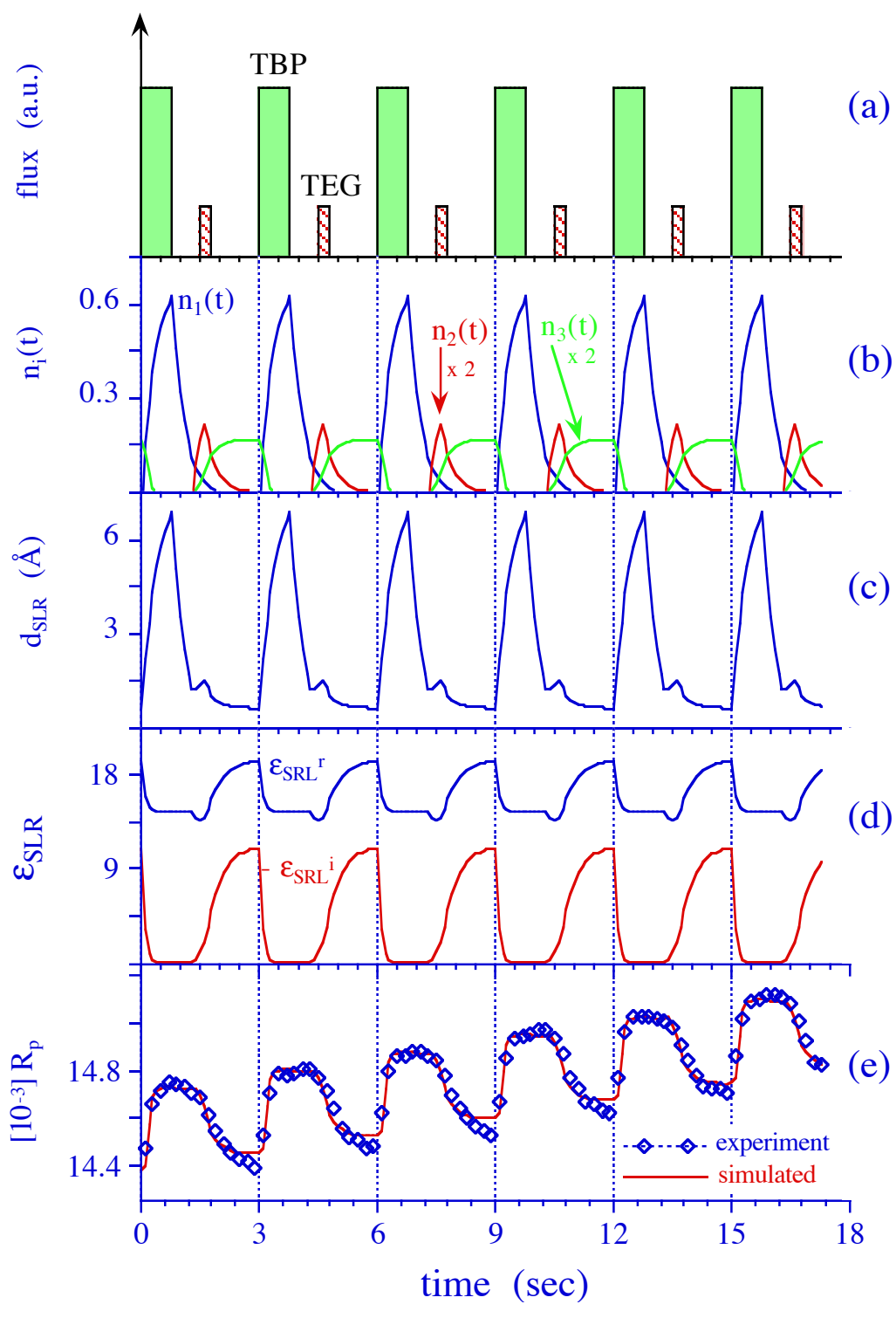

The values used in the calculations of the model are as follows. The molar volumes used in the minimization process were $\mathrm{V}_{\mathrm{TBP}}=128.6 \mathrm{~cm}^{3} \sum \mathrm{mol}^{-1}, \quad \mathrm{~V}_{\mathrm{TEG}}=148 \mathrm{~cm}^{3} \sum \mathrm{mol}^{-1}$, $\mathrm{V}_{1}=17 \mathrm{~cm}^{3} \Sigma \mathrm{mol}^{-1}, \quad \mathrm{~V}_{2}=13 \mathrm{~cm}^{3} \Sigma \mathrm{mol}^{-1}, \quad \mathrm{~V}_{3}=11.8 \mathrm{~cm}^{3} \Sigma \mathrm{mol}^{-1}, \quad \mathrm{~V}_{\mathrm{GaP}}=12.2 \mathrm{~cm}^{3} \Sigma \mathrm{mol}^{-1}$. 
The sticking coefficients used were $\beta_{\mathrm{TBP}}=0.0375$ and $\beta_{\mathrm{TEG}}=1.0$, and the effective SRL thickness parameter $\alpha_{\mathrm{SRL}}=0.75$. The geometrical parameter $\gamma=0.025$ was estimated in the minimization process. for a 2-inch diameter circular substrate wafer.

The numerical simulations were done using programs written in MATLAB code. The differential equations were solved numerically by the built-in function 'ode23', an adaptive mesh and low order Runge-Kutta method, and the optimization problem was solved using either a NelderMead algorithm ${ }^{20-22}$ or a Hooke-Jeeves procedure ${ }^{20}$. The authors are grateful to Prof. Kelley for providing us with the code "nelder" implementing the Nelder-Mead algorithm. In addition, D. Bortz provided us with the code "hj" implementing the Hooke-Jeeves procedure, which was used in some optimization calculations. We estimated the following parameter by averaging the results of independent best fits of experimental data sets where the TEG position and TEG flow was varied. The preliminary three-layer stack problem resulted in $\varepsilon_{3}=10.60-0.06 i$ and $\varepsilon_{4}=15.82-0.27 i$. Using these, the parameter estimation results from the four-layer stack model gave the following parameter values: the rate constants $\mathrm{k}_{1}=3.31, \mathrm{k}_{2}=1.55, \mathrm{k}_{3}=2.14, \mathrm{k}_{4}=0.052$, and $\mathrm{k}_{\mathrm{GaP}}=2.0 ;$ the optical responses $F_{1}=13.46-0.13 i, \quad F_{2}=13.56-0.0 i$ and $F_{3}=19.36-11.02 i$. These parameters produce an average SRL dielectric function of $16.82-4.47 i$ and an average film growth rate of $0.365 \AA$ Asec. An earlier study on the decomposition kinetics of TEG, analyzing the PR responses to a TEG exposure after growth interruptions ${ }^{12}$, gave for the rate constants $\mathrm{k}_{3}=2.5$ and $\mathrm{k}_{\mathrm{GaP}}=4.2$. The lower value for $\mathrm{k}_{\mathrm{GaP}}$ has been expected since the flow rates were lowered by about a factor of 2 in our experiments. 


\section{CONCLUSIONS}

We introduced a reduced order surface kinetic model using generalized reaction rate parameters to describe the decomposition kinetics of the organometallic precursors TBP and TEG used during heteroepitaxial growth of $\mathrm{GaP}$ on $\mathrm{Si}$. The set of coupled differential equations that describe the surface reaction kinetics provide information about the dynamics of molar concentrations of precursor fragments stored in the surface reaction layer and their incorporation into the underlying growing film. We fitted sets of experimental data using this model to identify the unknown parameters involved in the surface kinetics and their effect on the PR measurements. The results showed that the mathematical model can be used to effectively predict the large and small scale features of the experimental data and to model the deposition process. However, a validation of the predicted surface reaction layer constituents and their concentrations, as computed by the ROSK model, will require the development of highly surface sensitive, molecular specific diagnostic techniques that allow analysis of the dynamics in the SRL under steady-state growth. For this, the application of PRS in the infrared wavelength regime, using tunable laser sources, has been proposed.

\section{Acknowledgement}

The authors are indebted to Prof. K.J. Bachmann for helpful comments and suggestions, and to Prof. Kelley and D. Bortz (both at the Department of Mathematics, NCSU) for the computational support. Furthermore, we acknowledge the support for this work by DOD-MURI Grant F49620-95-1-0447. 


\section{References:}

1 Conf. Proc. of IPRM-9'97, ISSN 1092-8669 (1997).

2 Proceedings, 'DARPA Ultra Electronics \& Advanced MicroElectronics Program Review', Santa Fe, NewMexico, 26-31 Oct. 1997.

3 D. E. Aspnes and N. Dietz, "Optical Approaches for Controlling Epitaxial Growth", Applied Surface Science 130-132(1-4) pp. 367-376 (1998).

$4 \quad$ N. Dietz and K. J. Bachmann, "Real -time monitoring of epitaxial processes by parallelpolarized reflectance spectroscopy", MRS Bull. 20 (1995) p.49-55.

$5 \quad$ N. Dietz and K. J. Bachmann, "p-Polarized reflectance spectroscopy: a highly sensitive realtime monitoring technique to study surface kinetics under steady state epitaxial deposition conditions", Vacuum 47(2), pp. 133-40 (1996).

$6 \quad$ N. Dietz, N. Sukidi C. Harris and K.J. Bachmann, "Real-time Monitoring of Surface Processes by P-Polarized Reflectance", JVST A 15(3) p. 807-815 (1997).

$7 \quad$ N. Dietz, K. Ito, Real-time optical characterization of GaP heterostructures by p-polarized reflectance, Thin Solid Films 313-314(1-2) (1998) 615-620.

8 N. Dietz, A. Miller and K. J. Bachmann, "Real -time monitoring of homoepitaxial and heteroepitaxial processes by p-polarized reflectance spectroscopy", J. Vac. Sci. Technol. A 13, 153-155 (1995).

$9 \quad$ N. Dietz, A. Miller, J. T. Kelliher, D. Venables and K. J. Bachmann, "Migration-enhanced pulsed chemical beam epitaxy of GaP on Si(001)", Journal of Crystal Growth 150 (1-4) 691695 (1995).

10 N. Dietz, U. Rossow, D. Aspnes and K.J. Bachmann, "Real-Time Optical Monitoring of Epitaxial Growth: Pulsed Chemical Beam Epitaxy of GaP and InP Homoepitaxy and Heteroepitaxy on Si", JEM 24(11) 1571-76 (1995).

11 K. J. Bachmann, N. Dietz, A. E. Miller, D. Venables and J. T. Kelliher, "Heteroepitaxy of lattice-matched compound semiconductors on silicon", J. Vac. Sci. \& Technol. A 13, p.696704 (1995).

12 K. J. Bachmann, U. Rossow, N. Sukidi, H. Castleberry and N. Dietz, " Heteroepitaxy of Nearly Lattice-Matched Tetrahedrally Coordinated Compound Semiconductors on Si(100)", J. Vac. Sci. Technol. B 14, 3019 (1996) 
13 N. Dietz, U. Rossow, D. E. Aspnes and K.J. Bachmann, "Real-Time Optical Monitoring of Heteroepitaxial Growth Processes on Si under Pulsed Chemical Beam Epitaxy Conditions", Applied Surface Science, 102, p.47-51 (1996).

14 N. Dietz, N. Sukidi, C. Harris and K.J. Bachmann, "Real-time Characterization of the Optical Properties of an ultra-thin Surface Reaction Layer during Growth", Mat. Res. Soc. Symp. Proc. 441, 39-44 (1997).

15 K.J. Bachmann, N. Sukidi, N. Dietz, C. Hoepfner, S. LeSure, H.T. Tran, S. Beeler, K. Ito and H.T. Banks, "Real-time monitoring of the kinetics of pulsed chemical beam epitaxy by p-polarized reflectance", J. Cryst. Growth 183, 323-337 (1998).

16 N. Dietz, N. Sukidi, C. Harris and K.J. Bachmann, " Real-time Monitoring of Heteroepitaxial GaxIn1-xP/GaP Growth by P-Polarized Reflectance", Conf. Proc. of IPRM-9'97, ISSN 1092-8669, p. 521 (1997).

17 K. J. Bachmann, C. Hoepfner, N. Sukidi, A.E. Miller, C. Harris, D. E. Aspnes, N. Dietz, H.T. Tran, S. Beeler, K. Ito, H. T. Banks and U. Rossow, "Molecular Layer Epitaxy by Real-Time Optical Process Monitoring", Appl. Surf. Sci. 112, 38-47 (1997).

18 O. S. Heavens, "Optical Properties of Thin Solid Films", (Butterworths, London, 1955).

19 G. Burns, "Solid State Physics", Academic Press, Orlando, (ISBN: 0121460703) p. 461 (1985).

20 C. T. Kelley, "Iterative Methods for Optimization", North Carolina State University, Center of Research in Scientific Computation, to be published by SIAM (19999).

21 C. T. Kelley, "Detection and Remediation of Stagnation in the Nedler-Mead Algorithm using sufficient Decrease Condition", North Carolina State University, Center of Research in Scientific Computation, CRSC-TR97-2 (1997).

22 D. M. Brotz and C. T. Kelley, "The Simplex Gradient and Noisy Optimization Problems", in 'Computational Methods in Optimal Design and Control', J.T. Borggaard, J. Burns, E. Cliff and S. Schreck (ed.), (Progress in Systems and Control Theory), Birkhäuser, Boston, pp 7790 (1998). 